Research Article

\title{
Resumption of Meiotic Maturation of Oocytes, Pre- and Post-Implantational Embryonic Mortality under Conditions of Experimental Glomerulonephritis and Treatment of Silver Nanoparticles
}

\author{
Taras Viroslavovich Blashkiv ${ }^{\complement}$, Maria Sergeeevna Stupchuk, Valentine Aleksandrovna Sribna, \\ Oksana Mykolajivna Kalesnykova, Nataliya Georgejivna Grushka, Tetyana Yurievna Voznesenska
}

Department of Immunophysiology, O. O. Bogomoletz Institute of Physiology National Academy of Sciences of Ukraine, Kyiv, Ukraine.

$\triangle$ Corresponding author. E-mail: tblashkiv@gmail.com; Tel.: +38 (044) 256-20-83

Received: Mar. 9, 2018; Accepted: Aug. 16, 2018; Published: Nov. 2, 2018.

Citation: Taras Viroslavovich Blashkiv, Maria Sergeeevna Stupchuk, Valentine Aleksandrovna Sribna, Oksana Mykolajivna Kaleynykova, Nataliya Georgejivna Grushka, and Tetyana Yurievna Voznesenska, Resumption of Meiotic Maturation of Oocytes, Pre- and Post-Implantational Embryonic Mortality under Conditions of Experimental Glomerulonephritis and Treatment of Silver Nanoparticles. Nano Biomed. Eng., 2018, 10(4): 355-36I. DOI: 10.5101/nbe.v10i4.p355-361.

\begin{abstract}
The aim of the current study was to estimate the resumption of meiotic maturation of oocytes, preand post-implantation mortality of embryos under conditions of experimental glomerulonephritis and intravenous treatment of silver nanoparticles in mice. Experimental glomerulonephritis in mice was achieved by immunization of white laboratory mice of the first generation with a kidney antigen suspension derived from a parent. The treatment was carried out in the following way: Kidney antigen suspension - intraperitoneal three times 1 time per day; the procedure was repeated in 3 weeks, one time intraperitoneally with the same dose $(10 \mathrm{mkL}$ of suspension per 10 grams of body weight of the animal). Silver nanoparticles (AgNPs, $30 \mathrm{~nm}$ ) - intravenous treatment three times: 1 time per day for 1 $\mathrm{h}$ before immunization of animals with suspension of kidney antigen; as well as in 3 weeks once with the same dose $(2 \mathrm{mg} / \mathrm{kg})$. Thus, the number of oocytes that resumed meiosis in vitro from animals under experimental glomerulonephritis decreased as compared to the numbers in control animals; no inhibition of meiotic resumption of oocytes of animals was established under conditions of AgNPs treatment; the AgNPs treatment under conditions of experimental glomerulonephritis increased the number of oocytes that resumed meiosis in vitro compared with such values in the control and under conditions of experimental glomerulonephritis. The value of post-implantation embryonic mortality increased under conditions of experimental glomerulonephritis; no infertility of implantation in females of mice under conditions of AgNPs treatment; the AgNPs treatment under conditions of experimental glomerulonephritis decreased post-implantation mortality of embryos.
\end{abstract}

Keywords: Oocytes; Embryos; Silver nanoparticles

\section{Introduction}

Nowadays, biomedical nanotechnology develops rapidly in the search for new drugs. Among them drugs based on silver nanoparticles (AgNPs) occupy the leading position. The AgNP product industry focuses on a wide range of antimicrobial activity of AgNPs, including textiles, food storage containers, antiseptic 
sprays, catheters and bandages. Recently, AgNPs have become significant due to their therapeutic potential, which includes their ability to perform a role of antitumor agents [1]. Despite the AgNPs promising potential in medicine, the impact of AgNPs on human health (both positive and negative) is still not fully understood.

Glomerulonephritis, of immune etiology in particular, is a serious problem for women's reproductive health. There is evidence of a significant percentage of preterm labor and perinatal fetal loss among patients with membranous glomerulonephritis and IgA-glomerulonephritis. Moreover, according to the existing data, $90 \%$ of women with membranous glomerulonephritis gave birth to healthy children [2-4].

Studies that assess the effect of different doses and the multiplicity of the introduction of various sizes of AgNPs on reproduction of female animals gain topicality. Such studies will provide new data that will contribute to a fuller understanding of AgNPs mechanisms of action in the laboratory as well as provide a successful transition of silver nanotechnology into the clinics. Hence, the effect of AgNPs on mammalian cells and tissues requires further research. At present, in academic literature there are no data on the effect of AgNPs treatment has on meiotic maturation of oocytes and on pre- and postimplantational embryonic mortality under conditions of experimental glomerulonephritis.

The aim of the present study was to estimate resumption of meiotic maturation of oocytes, preand post-implantation mortality of embryos under conditions of experimental glomerulonephritis and intravenous treatment of silver nanoparticles in mice.

\section{Experimental \\ Materials \\ Animals}

Experiments (two series) were conducted on white laboratory mice: 64 females (10 weeks, 20-22 g) and 12 males in compliance with all requirements for work with laboratory animals (International European Convention for the Protection of Vertebrate Animals, Strasbourg, 1986). After the experiments, the animals anesthetized by Nembutal were exterminated by cutting the spinal cord.

\section{Characterization of nanoparticles}

AgNPs: Size - $30 \mathrm{~nm}$ in diameter; concentration - 8 $\mathrm{mg} / \mathrm{mL}$ for metal; shape - spherical; color - brown.

Reagents used for synthesis: Silver nitrate $\left(\mathrm{AgNO}_{3}\right)$ (BioXtra, > 99\%, titration, Sigma-Aldrich); carbonate potassium $\left(\mathrm{K}_{2} \mathrm{CO}_{3}\right)(99,995 \%$ trace metals basis, Sigma-Aldrich); Tannin (ACS reagent, Sigma-Aldrich) synthesized at the Ovcharenko Institute of Biocolloidal Chemistry NAS of Ukraine in accordance with the original protocol (by chemical condensation).

\section{Experimental glomerulonephritis in mice}

Experimental glomerulonephritis in mice was achieved by immunization of white laboratory mice of the first generation with a kidney antigen suspension derived from a parent.

Animal immunization was carried out at the rate of $10 \mathrm{mkL}$ of suspension per $10 \mathrm{~g}$ of body weight according to the following scheme: 3 times intraabdominal 1 time per day; re-immunization was carried out after 3 weeks with a single intraabdominal treatment of the same dose.

Before the start of and during the experiment, the animals were assessed by the objective status (appearance, general motor activity, need for food and water, 2 times a week, determined body weight) and the excretory kidney function (based on the number of spontaneous urinary tract disorders per day). Urine samples were determined using protein strips using a single dose of urine (diagnostic test strips for fast detection of protein, "Pharmaco", Ukraine).

\section{Methods}

The treatment was carried out in the following way:

Kidney antigen suspension - intraperitoneal three times 1 time per day; the procedure was repeated in 3 weeks, one time intraperitoneally with the same dose (10 mkL of suspension per 10 grams of body weight of the animal).

AgNPs - intravenous treatment 3 times: 1 time per day for 1 hour before immunization of animals with suspension of kidney antigen; as well as in 3 weeks once with the same dose $(2 \mathrm{mg} / \mathrm{kg})$.

\section{First series}

The effect of AgNPs on the resumption of meiotic maturation of oocytes (dissolution of the germinal vesicle) was investigated; the oocytes with atypical morphology (unevenly granulated cytoplasm and signs of fragmentation of the latter) were counted. 
Groups of animals: I - control animals ( $\mathrm{n}=$ 6), treated with physiological solution $(0.3 \mathrm{~mL})$; II - animals under conditions of experimental glomerulonephritis (immunized with antigenic kidney suspension) ( $\mathrm{n}=8)$; III - animals under conditions of experimental glomerulonephritis (immunized with an antigenic kidney suspension) were treated with silver nanoparticles $(\mathrm{n}=8)$; and IV - animals under conditions of treatment with silver nanoparticles (2 $\mathrm{mg} / \mathrm{kg}, 0.3 \mathrm{~mL})(\mathrm{n}=6)$.

Materials for the study (ovaries) were taken under anesthetic anesthesia on the third day (after the last injection).

\section{Second series}

The effect of AgNPs on pre- and post-implantation embryo mortality was investigated.

Groups of animals: I - control animals $(\mathrm{n}=6(2 \times$ 3 animals)), treated with physiological solution (0.3 $\mathrm{mL}$ ); II - animals under conditions of experimental glomerulonephritis (immunized with antigenic kidney suspension) $(\mathrm{n}=12(4 \times 3$ animals $))$; III - animals under conditions of experimental glomerulonephritis (immunized with an antigenic kidney suspension) were treated with silver nanoparticles $(n=12(4 \times$ 3 animals)); and IV - animals under conditions of treatment of silver nanoparticles $(2 \mathrm{mg} / \mathrm{kg}, 0.3 \mathrm{~mL})(\mathrm{n}$ $=6(2 \times 3$ animals $))$.

One day after the last injection, males were planted to females in a ratio of $1: 3$. The experimental materials were collected (ovaries, tubes and uterus) under anesthetic anesthesia on 10/11 days after males were planted to female.

The study was completed (24/25 days after males were planted), with birth live pups in control and experimental groups of animals.

\section{Oocytes cultivation}

The oocytes were isolated mechanically from the ovaries of mice in a non-enzymatic way (without cumulus cells and in cumulus-oocyte-cell complexes) and units/one ovary were counted. Then, the oocytes from one group were collected and distributed into separate chambers, $10 \sim 20$ oocytes each. All control and experimental oocytes were cultured under the same condition: A sterile box, cameras with 0.4 $\mathrm{mL}$ culture medium - Dulbecco's modified Eagle's medium (DME) and $15 \mathrm{mM}$ 4-(2-Hydroxyethyl) piperazine-1-ethanesulfonic acid, N-(2-Hydroxyethyl) piperazine- $\mathrm{N}^{\prime}$-(2-ethanesulfonic acid) (HEPES), $\mathrm{Ca}^{2+}$ concentration of $1.71 \mathrm{mM}$, temperature $37^{\circ} \mathrm{C}$, duration $2 \mathrm{~h}$. Morphological study of oocytes was performed under a microscope - Microscope XS4120 MICROmed binocular (MBS-10) after $2 \mathrm{~h}$ of cultivation (\% of total). The oocytes which restored the meiotic maturation (resumption meiosis) and were at metaphase I stage (germinal vesicle break-down) and oocytes with atypical morphology (unevenly granulated cytoplasm and fragmentation characteristics of the latter) were counted.

\section{Pre- and post-implantation mortality of embryos in mice}

Female control and experimental groups crossed with intact males. Counted: A - number of live embryos; B - number of seats of resorption (number of dead embryos); B - number of corpora lutea of pregnancy. Indicators of pre- and post-implantation death was calculated using the formula:

$(\mathrm{B}-\mathrm{A}+\mathrm{B}) / \mathrm{B} \times 100 \%$;

and

$\mathrm{B} /(\mathrm{A}+\mathrm{B}) \times 100 \%$.

\section{Statistical analysis}

For the statistical analysis of the results, the software package Origin 8 Pro (OriginLab Corp., North., MA, USA) and spreadsheets Microsoft ${ }^{\circledR}$ Excel 2003 were used. Student's t-test was performed for continuous variables. $\mathrm{P}<0.05$ was considered statistically significant. The statistical analysis of the research results was conducted by using analysis of variance ANOVA followed by comparison of mean values between groups by Newman-Coles test using the statistic program-5 (program GraphPad Prism 5.0 (GraphPad Software, San Diego, USA)).

\section{Results and Discussion}

Resumption of meiotic maturation of oocytes under conditions of experimental glomerulonephritis and intravenous AgNPs treatment

During the experiment, the following results were obtained:

Reduction in the number of oocytes isolated from one ovary under conditions of experimental glomerulonephritis was up to $9.8 \pm 1.3$ units/one ovary $(\mathrm{p}<0.05, \mathrm{n}=4)$, compared to control values of 17.3 
\pm 0.6 units/one ovary; no decrease in the number of such oocytes was developed under the condition of AgNPs treatment (17.4 \pm 1.2 units/one ovary); and under the condition of experimental glomerulonephritis intravenous AgNPs treatment, the number of oocytes in the ovary increased to $15.0 \pm 0.8$ units/one ovary $(\mathrm{p}<0.05, \mathrm{n}=4)$ as compared to the control values and under the condition of experimental systemic autoimmune disorder.

A decrease was found in the number of oocytes that resumed meiosis (Metaphase I) in vitro from animals under experimental glomerulonephritis $(\mathrm{p}<0.05, \mathrm{n}$ $=4$ ), compared to that in animals of control group; no inhibition of meiotic resumptionof oocytes of animals was developed under the condition of AgNPs treatment; the AgNPs treatment under the condition of experimental glomerulonephritis increased the number of oocytes that resumed meiosis in vitro $(\mathrm{p}<0.05, \mathrm{n}=$ 4 ), compared with the values in the control group and under conditions of experimental glomerulonephritis (Table 1).

\section{Pre- and post-implantation mortality of embryos under conditions of experimental glomerulonephritis and intravenous AgNPs treatment}

Animals of all groups were pregnant on the $10^{\text {th }} / 11^{\text {th }}$ day after males were planted to female. The data about pre- and post-implantation mortality of embryos under conditions of experimental glomerulonephritis and AgNPs treatment are presented in Table 2.

During the experiment, the following results were gained:

An increase was found in the value of postimplantational embryonic mortality under conditions of experimental glomerulonephritis; no infertility of implantation was found in females of mice under conditions of AgNPs treatment; and AgNPs treatment under conditions of experimental glomerulonephritis decreased post-implantation mortality of embryos.

Experiments were completed on the 24/25 day after males were planted to females with a birth in the control group $6.0 \pm 1.0(\mathrm{n}=6)$ live pups, while in the group under conditions of experimental glomerulonephritis $3.0 \pm 1.0(n=12)$, in the control group under conditions of AgNPs treatment $7.0 \pm$ $1.0(\mathrm{n}=12)$ and in the group under conditions of experimental glomerulonephritis and AgNPs treatment $5.0 \pm 1.0(\mathrm{n}=12)$ live pups.

Thus, the number of oocytes that resumed meiosis in vitro from animals under experimental glomerulonephritis decreased as compared to the numbers in control animals; no inhibition of meiotic

Table 1 Resumption of meiotic maturation of oocytes under conditions of experimental glomerulonephritis and intravenous AgNPs treatment

\begin{tabular}{|c|c|c|c|}
\hline \multicolumn{4}{|c|}{ Resumption of meiotic maturation of oocytes (\%) } \\
\hline $\begin{array}{c}\text { I } \\
\text { control }\end{array}$ & $\begin{array}{c}\text { II } \\
\text { experimental glomerulonephritis }\end{array}$ & $\begin{array}{c}\text { III } \\
\text { experimental glomerulonephritis + AgNPs }\end{array}$ & $\begin{array}{c}\text { IV } \\
\text { AgNPs }\end{array}$ \\
\hline $76.4 \pm 3.2$ & $30.3 \pm 1.1 *$ & $58.47 \pm 4.23 * \#$ & $74.21 \pm 2.17$ \\
\hline
\end{tabular}

Note: $* \mathrm{p}<0,05$, the probability of differences in the average data experimental groups $(\mathrm{n}=8)$ compared with such values in the control group of animals $(n=6)$. $\# p<0,05$, the probability of differences in the average data experimental groups III $(n=8)$ compared with such values in the experimental group II of animals.

Table 2 Pre- and post-implantation mortality of embryos under conditions of experimental glomerulonephritis and AgNPs treatment

\begin{tabular}{|c|c|c|}
\hline \multicolumn{3}{|c|}{ Mortality of embryos } \\
\hline & Pre-implantation (\%) & Post-implantation (\%) \\
\hline I (control) & $2.76 \pm 0.74$ & $1.38 \pm 0.37$ \\
\hline $\begin{array}{c}\text { II } \\
\text { experimental glomerulonephritis }\end{array}$ & $2.88 \pm 0.43$ & $5.16 \pm 0.63 *$ \\
\hline $\begin{array}{c}\text { III } \\
\text { experimental glomerulonephritis +AgNPs }\end{array}$ & $3.24 \pm 0.53$ & $1.38 \pm 0.37 \#$ \\
\hline $\begin{array}{c}\text { IV } \\
\text { AgNPs }\end{array}$ & $2.76 \pm 0.74$ & $1.35 \pm 0.34$ \\
\hline
\end{tabular}

Note: ${ }^{*} \mathrm{p}<0,05$, the probability of differences in the average data experimental groups $(\mathrm{n}=12)$ compared with such values in the control group of animals $(n=6)$. \# $p<0,05$, the probability of differences in the average data experimental groups III $(n=8)$ compared with such values in the experimental group II of animals. 
resumption of oocytes of animals was developed under the condition of AgNPs treatment; AgNPs treatment under the condition of experimental glomerulonephritis increased the number of oocytes that resumed meiosis in vitro, as compared to such values in the control group and under the condition of experimental glomerulonephritis. The value of post-implantational embryonic mortality increased under the condition of experimental glomerulonephritis; no infertility of implantation was found in females of mice under the condition of AgNPs treatment; AgNPs treatment under the condition of experimental glomerulonephritis decreased post-implantation mortality of embryos.

\section{Analysis and discussion of results}

Intravenous (IV) treatment (injection) of silver can still provide valuable information about processes taking place with AgNPs in vivo which has to overcome the main barriers (like skin, lungs, gastrointestinal tract) when put into circulation for clinical purposes (for example, dressing materials, intravascular medical devices for diagnosis, drug delivery systems, etc.). Since this study was not designed to simulate scenarios of human exposure to avoid the limited systemic exposure due to the existence of cellular barriers in the skin, gastrointestinal tract and lungs, in order to assess the potential systemic toxicity, we used IV treatment of AgNPs.

Since a dose of $2 \mathrm{mg} / \mathrm{kg}$ body weight in mice is equivalent to the human dose of $0.81 \mathrm{mg} / \mathrm{kg}$ body weight, a dose of about 10 (9.73) mg was applied to a person of $60 \mathrm{~kg}$, according to the principles of conversion of doses animals to humans [5]. We chose the dose of $2 \mathrm{mg} / \mathrm{kg}$ body weight, since it did not exceed the doses used in previous IV studies nor caused significant side effects in animals [6-8]. There are data about IV treatment of AgNPs [9, 10]. And silver was found in the main organs after AgNPs administration done in different ways [11-12].

There is also evidence of distribution of silver in tissues after oral administration in rats [12]. Citrate-coated AgNPs (size: $7.9 \pm 0.95 \mathrm{~nm}$ by transmission electron microscopy ((TEM) diameter) were investigated on male rats. Silver in the liver, lungs and kidneys was also measured at 24 and $96 \mathrm{~h}$ after treatment. The highest concentration of silver regardless of its coverage and regarding all sizes of particles was found in spleen, liver, lungs, kidneys and brain $24 \mathrm{~h}$ after IV treatment. The largest share of silver that reached the blood was filtered by the liver and excreted through the gall. AgNPs accumulated in the liver, lungs and kidneys; the accumulated AgNPs were released into the blood stream. AgNP levels in the urine were extremely low compared to the levels in the feces. When rats were injected with AgNPs, these particles were also detected in feces at $24 \mathrm{~h}$ after treatment, which suggested bile secretion of AgNPs [6, 7].

The embryo development under the condition of nanoparticle treatment is in the focus of scientists' attention. It has been established that in-vitro AgNPs of $50 \mathrm{mM}$ inhibited pre-implantation development of mice embryos [13].

There are also data showing that AgNPs do not have any effect on mice embryos [14]. It has been established that the repeated oral administration of AgNPs at $\geq 100 \mathrm{mg} / \mathrm{kg} /$ day during pregnancy causes oxidative stress in the liver tissue, but does not cause changes in the development of embryo and fetus under the condition of administration of doses up to 1000 $\mathrm{mg} / \mathrm{kg} /$ day [15]. Previously, we obtained data that rates of embryonic mortality had not changed under the condition of a ten-time treatment of AgNPs (2 mg/ $\mathrm{kg}$ and $4 \mathrm{mg} / \mathrm{kg}$ ) [16]. In this work, an increase in the value of post-implantational embryonic mortality was registered under the condition of experimental glomerulonephritis; no infertility of implantation was found in females of mice under the condition of AgNPs treatment; AgNPs treatment under the condition of experimental glomerulonephritis decreased the postimplantation mortality of embryos.

In literature, there are data about the impact of nanoparticles on oocytes [17-19]. There have been published data about the effect of nanoparticles of gold, silver and gold-silver alloy, which were covered with bovine serum albumin (BSA) on in-vitro cumulus-oocytes-cell-complexes of pigs [19]. Meiotic maturation of oocytes was assessed after $46 \mathrm{~h}$ of cultivation in vitro in the presence of different types of nanoparticles. The maturity of the oocytes in this case was determined as the percentage of oocytes with the formed first polar body in 350 oocytes per group. The concentration of nanoparticles was $10 \mathrm{mg} / \mathrm{mL}$, and all particles were conjugated to BSA [18].

Previously, we showed that a ten-time AgNPs treatment $(2 \mathrm{mg} / \mathrm{kg}$ and $4 \mathrm{mg} / \mathrm{kg})$ resulted in inhibition of oocytes meiotic maturation in mice; a single- and five-time AgNPs treatment $(2 \mathrm{mg} / \mathrm{kg}$ and $4 \mathrm{mg} / \mathrm{kg}$ ) increased the number of apoptotic cells, while the ten- 
time AgNPs treatment resulted in an increase of the apoptotic and necrotic follicular cells surrounding oocytes [16]. Influence of the tretment of AgNPs (30 nm, $2 \mathrm{mg} / \mathrm{kg}$ ) was estimated on the DNA of the nuclei of thymus cells, lymph nodes cells and cells of follicular environment of the oocyte under the condition of experimental systemic autoimmune disorder (glomerulonephritis) in mice. Experimental systemic autoimmune disorder (glomerulonephritis) in mice was achieved by immunization of white laboratory mice of the first generation with a kidney antigen suspension derived from a parent. And it was established that DNA damage increased in the nuclei of thymus cells, lymph nodes and cells of follicular environment of the oocyte under experimental glomerulonephritis; the damage on DNA of cells of the thymus and lymph nodes increased under the condition of AgNPs tretment; DNA damage in nuclei of thymus cells, lymph nodes and cells of follicular environment of the oocyte decreased under conditions of experimental glomerulonephritis and tretment of AgNPs [20].

In this work, it was established that the number of oocytes that resumed meiosis (Metaphase I) in vitro from animals under experimental glomerulonephritis was smaller than that in control animals; no inhibition of meiotic resumption of oocytes of animals was established under the condition of AgNPs treatment; the AgNPs treatment under the condition of experimental glomerulonephritis increased the number of oocytes that resumed meiosis in vitro as compared to such values in the control group and under the condition of experimental glomerulonephritis.

Probably, the differential toxicity of nanosilver may be connected with different coatings that are often applied to the AgNPs surface to achieve a stabilizing effect [21]. In addition, all the characteristics of nanoparticles need to be considered, including size, shape, crystalline structure, aggregation, chemical composition, surface properties (surface charge, surface area), solubility and porosity. Further studies are needed for the elucidation of mechanisms of AgNPs influence on germ and somatic cells administering AgNPs of different sizes and coatings.

Despite the fact that the toxicity appears to be caused by oxidation and inflammation [22], it is still not entirely clear what is responsible for the toxic effects of silver: whether this is the form of nanoparticles [23], or whether this is silver ions alone during oxidation of the metal [24]. There is evidence that AgNPs influence the induction of oxidative damage, change the regulation of enzymes, which are responsible for removing free radicals, change regulation of genes expression, which are involved in apoptosis and dis-regulation of cellular structures involved in storage, detoxification and metabolism of metals in various organs [25, 26]. Silver ions are equally toxic as both metal particles containing 80\% silver and as pure AgNPs, indicating that at least their toxic potential is similar [18]. Silver after an intravenous administration is registered in the kidneys of experimental animals [6, 7, 27]. And at present, we had only one assumption that may be due to the specific action of nanosilver on the immune system, but at the moment there is no evidence of this; further research is needed to clarify the effects of nanosilver on immune cells.Together with the data we received, for the future raises identifying possible structures, cells, organelles of the kidney as targets for nanosilver, and the mechanisms responsible for the fact that AgNPs treatment under conditions of experimental glomerulonephritis improves reproductive function in female animals.

\section{Conclusions}

In mice females, the AgNPs treatment under conditions of experimental glomerulonephritis increased the number of oocytes that resumed meiosis in vitro and decreased post-implantation mortality of embryos. The given data are also one more proof to support the hypothesis that the AgNPs toxicity is primarily linked to $\mathrm{Ag}^{+}$bioavailability. It is important to stress that our data suggest that oocytes in mammals have the potential for the deployment of compensatory mechanisms of action under conditions of $\mathrm{Ag}^{+}$ administration at doses lower than those causing obvious toxicity.

\section{Acknowledgments}

We wish to thank Ovcharenko Institute of Biocolloidal Chemistry, National Academy of Sciences (NAS) of Ukraine and Ms. Ludmila Rieznichenko, $\mathrm{PhD}$ for providing the samples of AgNPs for this research.

\section{Conflict of Interests}

The authors declare that no competing interest 
exists.

\section{References}

[1] G. Braun, T. Friman, H. Pang, et al., Etchable plasmonic nanoparticle probes to image and quantify cellular internalization. Nat Mater, 2014, 13(9): 904-911.

[2] G. Malik, A. Al-Harbi, S. Al-Mohaya, et al., Repeated pregnancies in patients with primary membranous glomerulonephritis. Nephron, 2002. 91(1): 21-24.

[3] A. Al-Harbi, G. Malik, S. Al-Mohaya, et al., Antiglomerular Basement Membrane Antibody Disease Presenting as Acute Renal Failure During Pregnancy. Saudi J Kidney Dis Transpl, 2003, 14(4): 516-521.

[4] M. Segelmark, T. Hellmark, Autoimmune kidney diseases. Autoimmun Rev, 2010. 9(5): A366-371.

[5] S. Reagan-Shaw, M. Nihal, and N. Ahmad, Dose translation from animal to human studies revisited. FASEB $J, 2008,22(3)$ : 659-661.

[6] K. Park, E. Park, I. Chun, et al., Bioavailability and toxicokinetics of citrate-coated silver nanoparticles in rats. Arch Pharm Res, 2011, 34: 153-158.

[7] Y. Xue, S. Zhang, Y. Huang, et al., Acute toxic effects and gender-related biokinetics of silver nanoparticles following an intravenous injection in mice. J Appl Toxicol, 2012, 32: 890-899.

[8] W. De Jong, L. Van Der Ven, A. Sleijffers, et al., Systemic and immunotoxicity of silver nanoparticles in an intravenous 28 days repeated dose toxicity study in rats. Biomaterials, 2013, 34: 8333-8343.

[9] C. Rigo, L. Ferroni, I. Tocco, et al., Active silver nanoparticles for wound healing. Int J MolSci, 2013, 14(3): 4817-4840.

[10] L. Wei, J. Lu, H. Xu, et al., Silver nanoparticles: Synthesis, properties, and therapeutic applications. Drug Discov Today, 2015, 20(5): 595-601.

[11] M. van der Zande, R. Vandebriel, E. Van Doren, et al., Distribution, elimination, and toxicity of silver nanoparticles and silver ions in rats after 28-day oral exposure. ACS Nano, 2012, 6(8): 7427-7442.

[12] J. Kwon, A. Minai-Tehrani, S. Hwang, et al., Acute pulmonary toxicity and body distribution of inhaled metallic silver nanoparticles. Toxicol Res, 2012, 28(1): 25-31.

[13] P. Li, T. Kuo, J. Chang, et al., Induction of cytotoxicity and apoptosis in mouse blastocysts by silver nanoparticles. Toxicol Lett, 2010, 197(2): 82-87.

[14] U. Taylor, W. Garrels, A. Barchanski, et al., Injection of ligand-free gold and silver nanoparticles into murine embryos does not impact pre-implantation development. Beilstein J Nanotechnol, 2014, 21(5): 677-688.

[15] W. Yu, J. Son, J. Lee, et al., Effects of silver nanoparticles on pregnant dams and embryo-fetal development in rats. Nanotoxicology, 2014, 8(1): 85-91.

[16] A. Lytvynenko, L. Rieznichenko, V. Sribna, et al., Functional status of reproductive system under treatment of silver nanoparticles in female mice. International Journal of Reproduction, Contraception, Obstetrics and Gynecology, 2017, 6(5): 1713-1720.

[17] H. Johnston, G. Hutchison, F. Christensen, et al., A review of the in vivo and in vitro toxicity of silver and gold particulates: particle attributes and biological mechanisms responsible for the observed toxicity. Crit Rev Toxicol, 2010, 40(4): 328-346.

[18] D. Tiedemann, U. Taylor, C. Rehbock, et al., Reprotoxicity of gold, silver, and gold-silver alloy nanoparticles on mammalian gametes. Analyst, 2014, 139(5): 931-942.

[19] U. Taylor, D. Tiedemann, C. Rehbock, et al., Influence of gold, silver and gold-silver alloy nanoparticles on germ cell function and embryo development. Beilstein $J$ Nanotechnol, 2015, 5(6): 651-664.

[20] T. Voznesenskaya, M. Stupchuk, N. Grushka, et al., The influence of the tretment of silver nanoparticles on the DNA of the nuclei of thymus cells, lymph nodes and the follicular environment of the oocyte under conditions of experimental systemic autoimmune disorder. Bulletin of Problems Biology and Medicine, 2018, 1(143): 327-331 [in Ukrainian].

[21] N. Durán, M. Durán, M. de Jesus, et al., Silver nanoparticles: A new view on mechanistic aspects on antimicrobial activity. Nanomedicine, 2016, 12(3): 789799.

[22] Y. Hsin, C. Chen, S. Huang, et al., The apoptotic effect of nanosilver is mediated by a ROS- and JNK-dependent mechanism involving the mitochondrial pathway in NIH3T3 cells. Toxicol Lett, 2008, 179(3): 130-139.

[23] G. Laban, L. Nies, R. Turco, et al., The effects of silver nanoparticles on fathead minnow (Pimephalespromelas) embryos. Ecotoxicology, 2010, 19(1): 185-195.

[24] S. Chernousova, M. Epple, Silver as antibacterial agent: ion, nanoparticle, and metal. Angew Chem Int Ed Engl, 2013, 52(6): 1636-1653.

[25] J. Choi, S. Kim, J. Ahn, et al., Induction of oxidative stress and apoptosis by silver nanoparticles in the liver of adult zebrafish. Aquat Toxicol, 2010, 100(2): 151-159.

[26] J. Maillard, P. Hartemann, Silver as an antimicrobial: facts and gaps in knowledge. Crit Rev Microbiol, 2013, 39(4): 373-383.

[27] C. Recordati, M. De Maglie, S. Bianchessi, et al., Tissue distribution and acute toxicity of silver after single intravenous administration in mice: nano-specific and size-dependent effects. Part Fibre Toxicol, 2016, 29(13): 12.

Copyright $@$ Taras Viroslavovich Blashkiv, Maria Sergeeevna Stupchuk, Valentine Aleksandrovna Sribna, Oksana Mykolajivna Kaleynykova, Nataliya Georgejivna Grushka, and Tetyana Yurievna Voznesenska. This is an open-access article distributed under the terms of the Creative Commons Attribution License, which permits unrestricted use, distribution, and reproduction in any medium, provided the original author and source are credited. 